\title{
PRONY FILTERING OF SEISMIC DATA: MATHEMATICAL AND PHYSICAL MODELLING
}

\author{
Georgy M. Mitrofanov ${ }^{1}$ and Viatcheslav Ivanovich Priimenko²
}

\begin{abstract}
This article presents results of the Prony filtering method testing using mathematical and physical models. They illustrate features and capabilities of the proposed method of processing and analysing the recorded signals, which also include reflected seismic signals. Guidelines for choosing the optimal method parameters are demonstrated using examples. The use of such parameters provides the best extraction and parameters estimation of target signals. Based on them, it allows us to analyse the absorbing and scattering properties of the media for the local areas, for the analysed reflectors in particular.
\end{abstract}

Keywords: Prony transform, signal decomposition and filtering, dispersion and absorption of seismic energy, frequency-dependent effects.

RESUMO. Este artigo teve por objetivo apresentar e analisar os resultados da aplicação do método de filtragem de Prony em modelos físicos e matemáticos. Tais resultados ilustram as características e o potencial do método proposto no processamento e a análise de sinais, incluindo também os eventos sísmicos de reflexão. As diretrizes para escolher os parâmetros otimizados do método são demonstradas através de exemplos. 0 uso de tais parâmetros proporciona a melhor estimativa, e viabiliza a separação dos sinais alvo. Com base em tais sinais, é possível analisar as propriedades de absorção e dispersão dos meios em áreas locais, em particular, nos refletores analisados.

Palavras-chave: transformada de Prony, decomposição de sinal e filtragem, dispersão e absorção de energia sísmica, efeitos de frequência.

\footnotetext{
${ }^{1}$ Institute of Geology and Geophysics, Siberian Branch of the Russian Academy of Sciences, pr. Koptyuga, 4, Akademgorodok, 630090, Novosibirsk, Russia. Phone: (73832) 333909 - E-mail: george@uiggm.nsc.ru

2Laboratory of Petroleum Engineering and Exploration, North Fluminense State University Darcy Ribeiro, Rod. Amaral Peixoto, km 163, Av. Brennand, s/n, Imboassica, 27925-535 Macaé, RJ, Brazil. Phone: +55(22) 2765-6571; Fax: +55(22) 2765-6577 - E-mails: slava@lenep.uenf.br; slava211054@gmail.com
} 


\section{INTRODUCTION}

The paper (Mitrofanov \& Priimenko, 2011) provides a general description of the Prony filtering method and seismic data process technology, built on its basis. The given examples and results of the test experiments mainly refer to the intrinsic properties of the Prony transform and Prony filtering algorithms when used for the analysis of seismic signals. Many issues related to the features of the entire filtering process weren't considered in the paper. At the same time, the study of such features is an important element in the development of the method in view of its non-linearity. That is why various test experiments were carried out for quite a long time since the first studies on the application potential of the method (Mitrofanov et al., 1999).

This paper presents the results of the method testing on two types of model experiments. The first type is the mathematical modelling of wave fields in the case of massive gas deposits. The wave fields calculated for a rather simple media model are complicated with the noise taken from real data. The second type is associated with a two-dimensional physical modelling. The purpose of the experiments was to determine the capabilities and features of the application of Prony filtering when processing seismic signals close to real ones. The use of physical models not only allowed studying the properties of Prony filtering, but also clarifying the range of problems which can be resolved applying the proposed method. In particular, they showed which features of the seismic signals behaviour at high frequencies can be determined and what features of the media structure they can correspond to. Thus, in contrast to the previous model experiments, this level of testing allows us to get closer to handling of real seismic data presented in the form of time sections. As a consequence, there is a need to discuss the methods of using Prony filtering for relevant materials, which leads to consideration of the question of the optimal choice of the parameters which improve functionability of the method.

During the test experiments considerable attention has been paid to noise immunity of the filtering procedure as well as to the possibilities of its use for the allocation of objects of interest and to the analysis of the characteristics of the media under study. The following aspects have been studied in great detail:

- stable extraction of weak seismic signals which are overlapped by other wave components with a greater energy formation;

- possible change in the waveform due to application of nonlinear transformation to which the method under consideration is related;

- comparison of the results of Prony and band-pass filtering, as the most similar traditional procedure of the same orientation;

- the technology for optimal selection of the filtering parameters, providing the best extraction of the target signals.

\section{MATHEMATICAL MODELLING}

\section{Description of the model and data}

The considered mathematical model is the sum of the two wave patterns. Total time section corresponding to the media model, shown in Figure 1, was taken as the first wave pattern. During its composition we used synthetic 48-channel seismograms, which were calculated on the basis of the ray method and the edge waves method in the region of the diffracting point (Klem-Musatov \& Aizenberg, 1984). The model was proposed by Girshgorn, JSC 'Yamalgeofizika', Russia. Its basis was the real gas deposit related to the Cenomanian deposits. The model contains four layers, the thickness of which is specified in the figure. The third layer, which was between the borders of hll and hlll, pinches out at the point with the horizontal coordinate $x=3 \mathrm{~km}$, which is the main feature of the model.

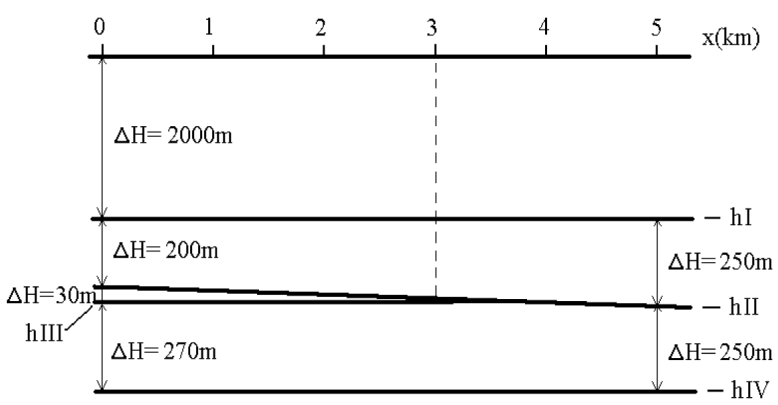

Figure 1 - Model of the medium used to calculate the synthetic seismograms.

A part of the real time section shown in Figure 3(a), was considered as the second wave pattern. It served as a noise component relative to the synthetic time section, built for the specified media model. Adding this component to the total wave field with different amplitude levels allowed analysing the noise immunity of the considered filtering procedure for different signal-to-noise ratio.

When constructing model seismograms we used the wavelet, which corresponded to the sum of two different Ricker wavelets with the frequency of 40 and $80 \mathrm{~Hz}$ respectively. The $40 \mathrm{~Hz}$ frequency pulse was considered as the main component of the simulated wave field, and the $80 \mathrm{~Hz}$ frequency pulse was the target signal. The ratio of the target signal amplitude and the main component amplitude was one to five. Our experience shows that such ratio of main and high-frequency components is typical for real data. 
Thus, the trace corresponding to the simulated time section can be expressed by the expression:

$$
\begin{gathered}
y(x, t)= \\
S^{(40)}(x, t)+0.2 \cdot S^{(80)}(x, t)+a^{(n)} \cdot n(x, t),
\end{gathered}
$$

in which $x, t$ are the spatial and temporal coordinates and $S^{(f)}(x, t)$ corresponds to the time section, obtained from model seismograms built based on the Ricker wavelet in the following form:

$$
S^{(f)}(t)=\left(1-2(\pi f t)^{2}\right) \cdot e^{-(\pi f t)^{2}}
$$

with the set frequency $f$. The $n(x, t)$ function describes additive noises, which are included in the simulated data with a ratio of $a^{(n)}$ (we shall discuss the peculiarities of the noise below). Meanwhile, the first two sums define the signal part of the model and the value of $a^{(n)}$ determines the signal-to-noise ratio for the simulated data.

The use of the specified signal structure allowed investigating of these questions within a single model experiment:

- studying characteristics of a wave field that corresponds to the elements of the media with small angles of pinching for target horizons, which is typical for Western Siberia areas;

- exploring the method ability for extraction of highfrequency components of the field overlapped by lowfrequency components with high-energy;

- analysing stability and noise immunity of the resulting estimates of signal components in presence of the noise of different levels, which are close to the real data in terms of characteristics.

Note that the Ricker wavelet, possessing a wide range of the Prony spectrum (see Mitrofanov \& Priimenko, 2011), complicates the task of separating the low-frequency and high-frequency components. Therefore, in these experiments it was possible to assess how well signals of this type can be recovered during Prony filtering.

The analysed parts of the constructed synthetic sections $S^{(40)}(x, t)$ and $S^{(80)}(x, t)$ are presented in the left side of Figures 2(a) and 2(b). They contain reflections of longitudinal waves from all horizons of the media model. In the sections construction was made the time shift on $200 \mathrm{~ms}$. Therefore, to obtain the real time it is necessary to increase the presented time by $200 \mathrm{~ms}$. This is not a matter of principle for conducted experiments, but permits us identify more precisely these reflections. On the right side of the same figure we showed the results of spectral analysis. During the spectral analysis were calculated the values of the amplitude spectra and energy for trace intervals with the width $100 \mathrm{~ms}$, which contained reflections of the horizons hll and hlll. The maximum values of the amplitude spectra correspond to light tones in the figures.

Comparison of the wave fields presented in Figures 2(a) and 2(b) shows that, based on the component of $S^{(80)}(x, t)$, reflection arrival times from all the horizons are defined more precisely. The structure of the reflected signals, as well as changing of the amplitude and geometry of the reflections from the hll and hlll horizons, points out that in the absence of noise both wave patterns allow mapping out the area of the pinching out of the target layer. However, if the frequency is $40 \mathrm{~Hz}$ this area can only be determined approximately. At the frequency of $80 \mathrm{~Hz}$ the determination accuracy of the pinching zone increases significantly both in the wave picture and energy graph. It also should be mentioned that the spectral composition of the analysed reflections changes significantly. For a pulse with a frequency of $80 \mathrm{~Hz}$ it becomes much wider and has sufficiently high values of the amplitude spectrum in the frequency range from $25 \mathrm{~Hz}$ to $100 \mathrm{~Hz}$.

The time section and the results of spectral analysis corresponding to the full signal component of the expression (1), which includes both the Ricker wavelets, is shown in Figure 2(c). It is easily understood that the presence of a signal component with a lower frequency and significantly higher energy overrides all the effects related to high frequency. In principle, the amplitude spectrum shows that in this case, the signal has more high-frequency components than it had in the case of one pulse with a frequency of $40 \mathrm{~Hz}$. However, a small value of these components does not give them the opportunity to emerge significantly in the time and energy concepts.

Figure 3 shows the information about the noise component and the simulated time section $y(x, t)$ when $a^{(n)} \neq 0$. The spectral analysis for all time sections shown in this figure was carried out in large time intervals of $700 \mathrm{~ms}$, as is usually done in the real data processing when the arrival times of the target signals are not known. It shows that the spectrum of the time section acting as a noise contains frequencies up to $35 \mathrm{~Hz}$, and the main frequencies are concentrated in the area of $25 \mathrm{~Hz}$ (see Fig. 3(a)). This feature of the spectra can be quite typical for many regions where surface seismic surveys are conducted. As a rule, the results of such studies include intensive reflections with frequencies of 15 to $40 \mathrm{~Hz}$, covering high-frequency signals which are present in the observed wave field.

The examples of the simulated data are shown in Figures $3(b)$ and 3(c). They were obtained by combining the wave fields presented in Figures 2(c) and 3(a) with different values of $a^{(n)}$. In the case presented in Figure $3(\mathrm{~b})$ the value of was selected in order to provide a signal-to-noise ratio close to 3 in the area 


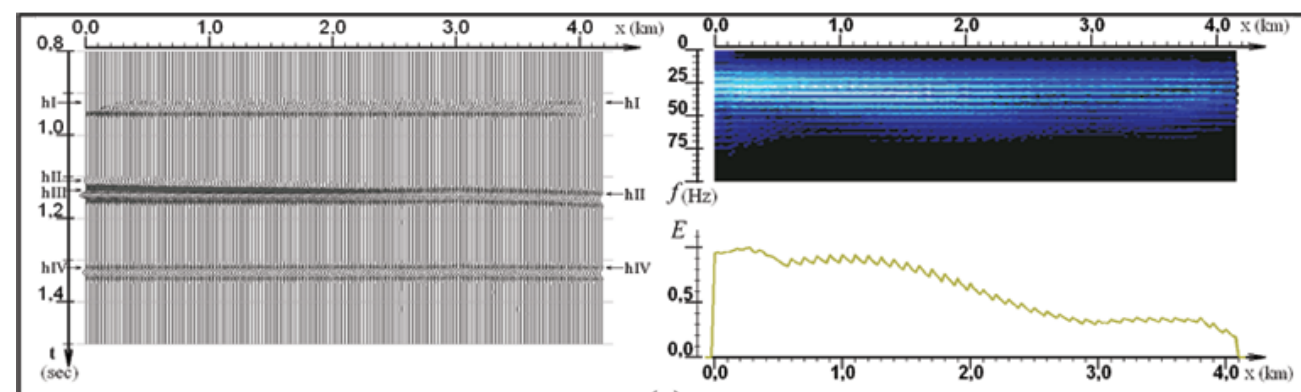

(a)

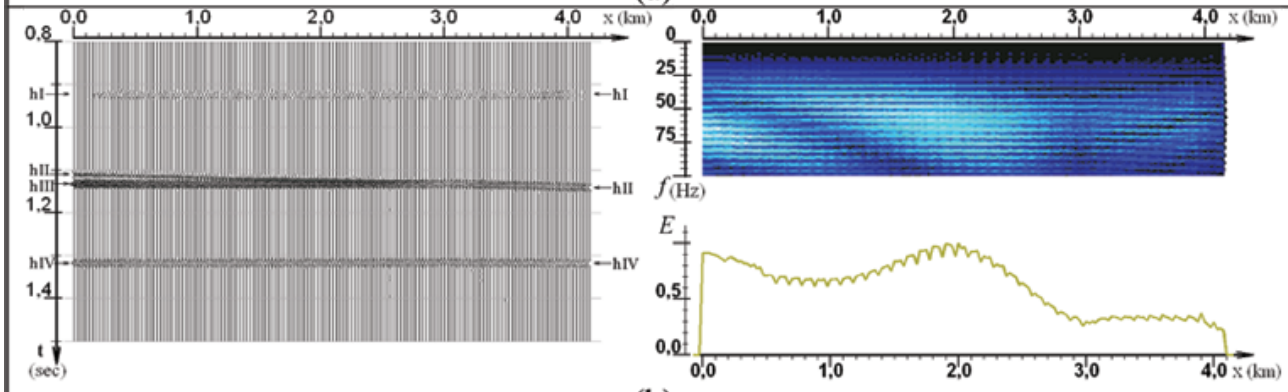

(b)

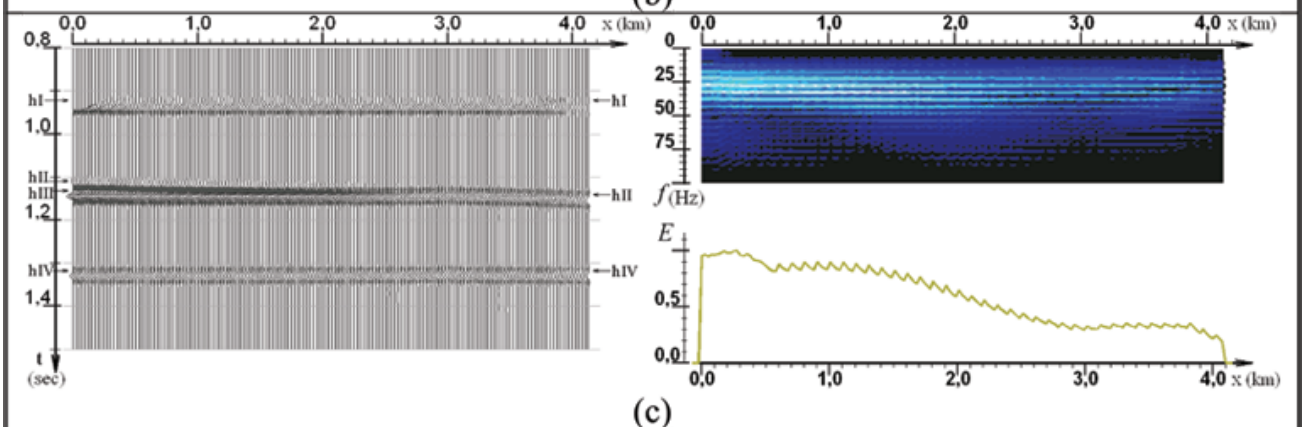

Figure 2 - Synthetic time sections (left column) and the results of their spectral analysis (right column) (explanation in text).

where reflections from the hll horizon are available. In the second case the value of this ratio was close to 1 , which can be considered as typical when processing real data. The presented figures show that in case of a rather high signal-to-noise ratio (greater than 2) the reflected signals from the main horizons can be easily traced, but the information about the pinching-out zone is rather inaccurate. A marked change in energy along the profile associated with the pinching-out of a layer can be observed only at relatively high signal-to-noise ratio. According to the results of spectral analysis we found that frequencies up to $50 \mathrm{~Hz}$ are the most significant in the amplitude spectrum of the simulated data. At the highest frequencies there are only relatively minor manifestations that indicate the presence of high-frequency components in the wave field under consideration. Such behaviour of the amplitude spectra appears to us as typical for real seismic experiments.

Spectra and energy distribution along the profile corresponding to the experiment with a signal-to-noise ratio equal to 1 (see
Fig. 3(c)) are most similar to those dependencies, which are observed in the real data. This means that there are stable components (manifested in our representation in the form of light stripes in the space coordinate) up to frequencies of $45-55 \mathrm{~Hz}$ in the amplitude spectra of the analysed time interval. At the frequency of $70-80 \mathrm{~Hz}$ spectra are generally of "spotty" nature. The frequency of $60-65 \mathrm{~Hz}$, in which it is possible to obtain robust estimates of the signal components, is the most interesting part of the highfrequency spectrum for land seismic prospecting.

\section{The analysis of the Prony filtering signal features and results on the example of one model trace}

Before turning to the processing of the time sections we shall consider several experiments dealing with the separation of a frequency component of a signal related to $S^{(80)}(x, t)$. This will allow a better understanding of the filtering process applied to the whole set of traces of $y(x, t)$. 


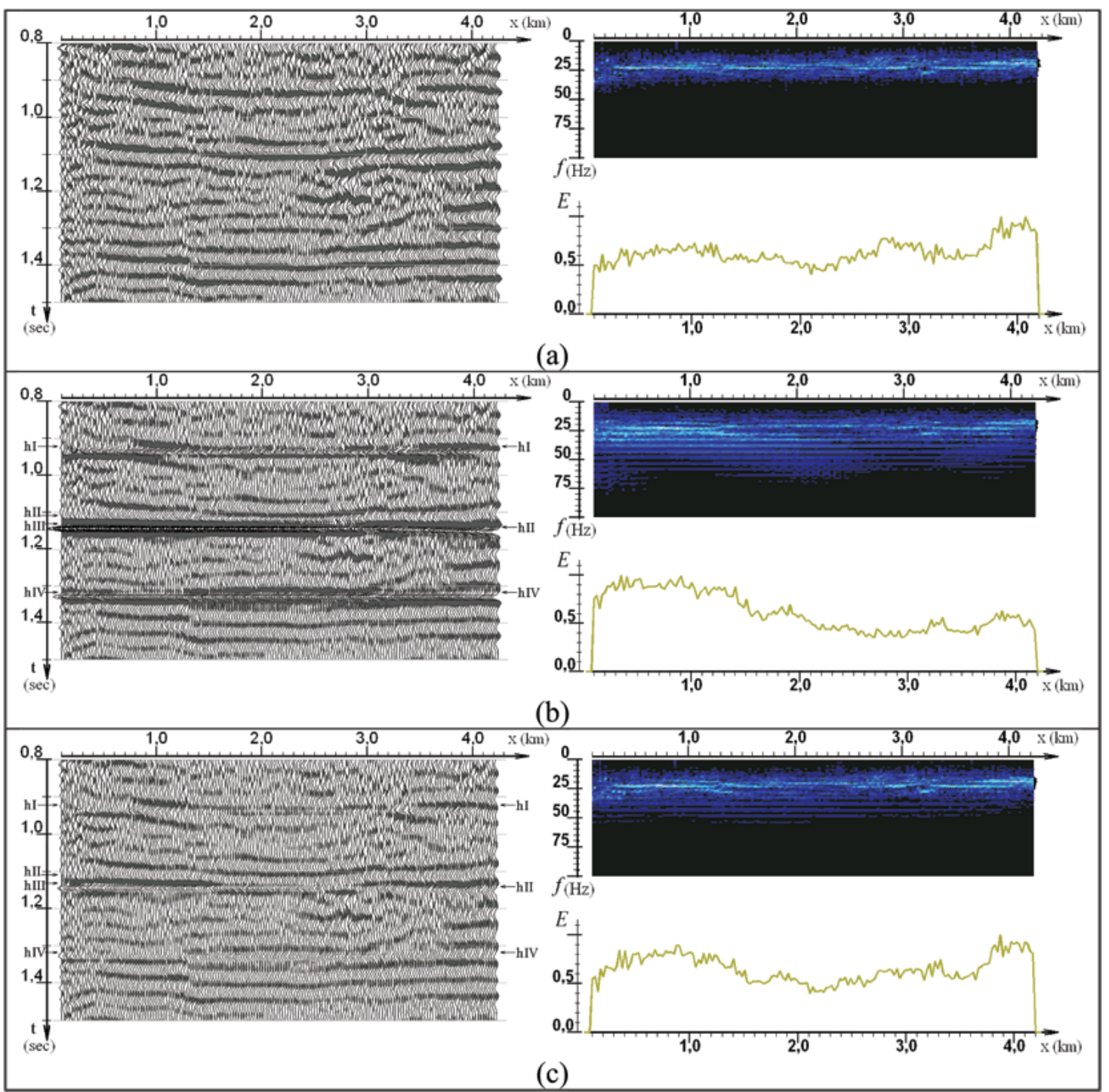

Figure 3 - Noise component and full time sections (left column) with the results of spectral analysis (right column) (explanation in text).

The relevant experiments were carried out for the trace with $x=0.5 \mathrm{~km}$. The trace selection was not of fundamental importance. But for the trace, located at the mentioned selection, there were several good points. In its case there were no edge effects particularly manifested in the reflection of hl horizon (see Fig. 2). In addition, there is still a clear separation of the reflections of hll and hIII horizons for this position on the profile at a frequency of $80 \mathrm{~Hz}$. That is why we can estimate the temporal resolving ability of Prony filtering. In the experiments we used a trace without noise, i.e., $a^{(n)}=0$, which made it possible to study possible changes in the signals that are directly related to the effect of the filtering process. The view of tested trace for the three views of Figure 2 is shown in Figure 4. When depicting the full signal com- ponent of the model (1) the included component of $S^{(80)}(x, t)$ is highlighted in black. It provides a better understanding of the relationship between separated components.

Before turning to the issues of separation of signals, we shall consider what type of distinct Prony spectra

$$
\Re_{\tau, T}(M)=\left\{A_{k}, \alpha_{k}, f_{k}, \theta_{k}\right\}_{k=1}^{k=M}
$$

can be obtained for the analysed signals when choosing different durations of the time interval $T$. Here $A_{k}, \alpha_{k}, f_{k}, \theta_{k}$ are the values of the Prony expansion parameters (respectively, the amplitude, damping, frequency and phase) and $\tau$ determines the position of the interval. The importance of this analysis was pointed out in the paper (Mitrofanov \& Priimenko, 2011). 


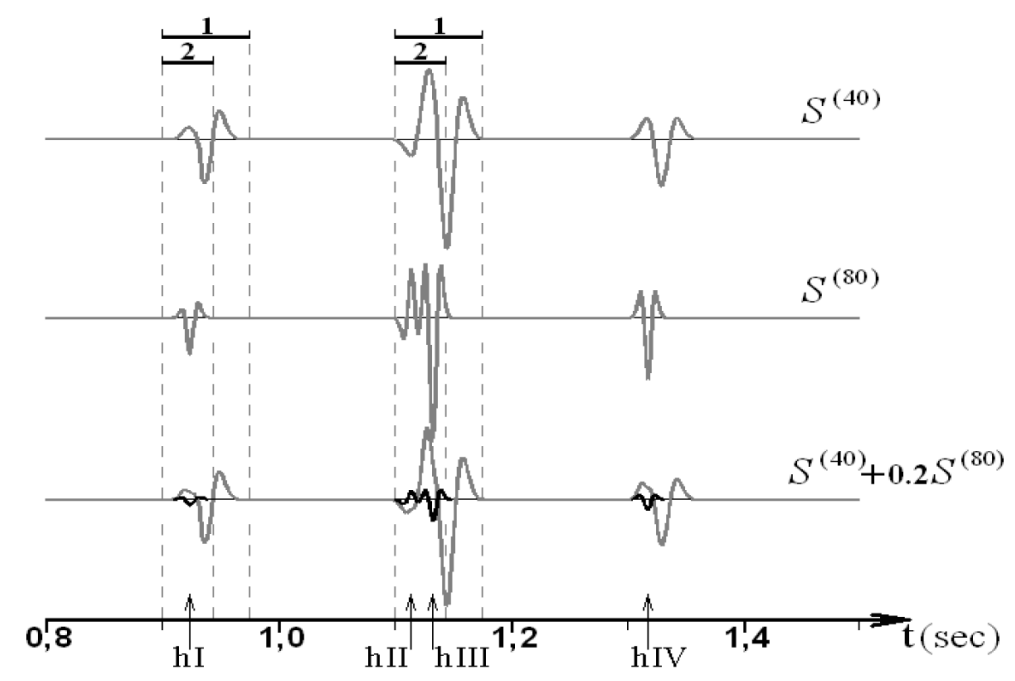

Figure 4 - Trace of synthetic time section with the coordinate $x=0.5 \mathrm{~km}$ for different signals.

As an example we consider two intervals: (i) with duration of $0.076 \mathrm{sec}$, and (ii) with duration of $0.044 \mathrm{sec}$. The position of the respective intervals relative to the analysed signals is shown in Figure 4. The duration of the interval (i) for a given location allows the incorporation of any of the considered signals. The duration of interval (ii) is sufficient enough only for signals of $S^{(80)}(x, t)$.

The Prony spectra obtained for signals falling in the range of (i) and (ii) are shown in Figure 5. At the same time it shows the values of only three of the four Prony parameters as the value of the phases were of no interest. The figure uses a variety of colours for the three types of signals: green for $S^{(40)}$, black for $S^{(80)}$ and red for $S^{(40)}+0.2 \cdot S^{(80)}$. Figures $5(\mathrm{a})$ and $5(\mathrm{c})$ show the spectra of signals that fall within the range of (i) with the beginning at $0.9 \mathrm{sec}$ and $1.1 \mathrm{sec}$, respectively, and Figures $5(\mathrm{~b})$ and $5(\mathrm{~d})$ present the spectra for the interval of (ii) with the same time start.

Several conclusions can be made from the analysis of the structure of the specified spectra. The simplest is that the length of the interval determines the order of approximation model $M$. The longer the duration of the interval, the greater number of components in the Prony expansion is required to be determined to ensure the required accuracy of approximation of the analysed signal (in the experiments, this accuracy made up 0.01 of the maximum amplitude of the signal). Next conclusion concerns a high degree of similarity of frequency parameter values, which are defined for different signals in different intervals. Therefore in decomposition of the signal $S^{(40)}$ there are high frequency components, and in $S^{(80)}$ the low frequency components are present. Also, a wide variation of the frequency value from $10 \mathrm{~Hz}$ to $150 \mathrm{~Hz}$ is typical for these values. As it was already mentioned, this is the Ricker wavelet feature that brings some difficulties in the filtering procedure. Despite the high degree of similarity in the values of the frequency, a joint analysis of the frequency and damping values related to the same Prony components shows the following. High frequencies values, which were obtained for signal $S^{(80)}$, meet the large absolute values of the damping parameter. This is an opportune moment for the Prony filtering, because it is based on a joint analysis of frequencies and damping values.

Figure 6 shows in black the results of filtering performed when determining the $80 \mathrm{~Hz}$ component. The results shown in Figure 6(a) refer to the area where the reflection from the hl horizon is available. They were obtained using the values of the Prony spectra, which are presented in Figures 5(a) and 5(b) in red, i.e., for $S^{(40)}+0.2 \cdot S^{(80)}$. In the filtering process, the values of the frequencies are in the range of $[70,100] \mathrm{Hz}$ and damping of $[-200,200]$. We see that in both cases it is possible to select the target component. But in case of a wider interval (1) it is determined worse and as a result approximates the original signal with less precision. A similar situation exists for the interference signal with the reflection of the hll and hlll horizons (Fig. 6(b)).

An even greater difference in the defined components is produced when according to the Prony filtering technology a moving time interval is used for the full trace. Figure $6(c)$ shows the results obtained by moving the considered intervals. When using an interval of longer duration the shape of the target signal is restored worse and distortions of a shape are often observed, which are associated with a combination of filtering results obtained at different positions of the interval. For intervals of shorter duration in the case of determining the component of $80 \mathrm{~Hz}$ there 

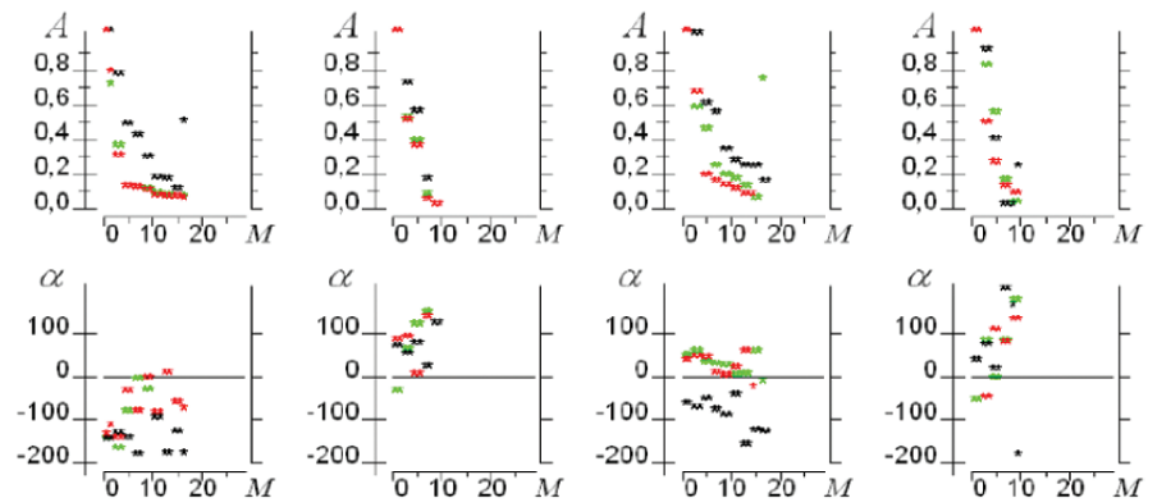

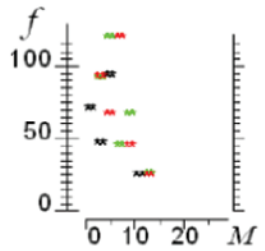

(a)

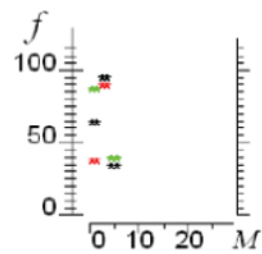

(b)

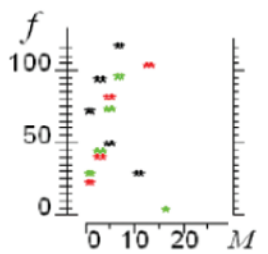

(c)

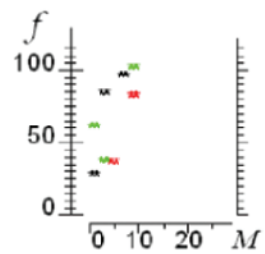

(d)

Figure 5 - Prony discrete spectra calculated for the signals corresponding to considered time intervals (explanation in text).

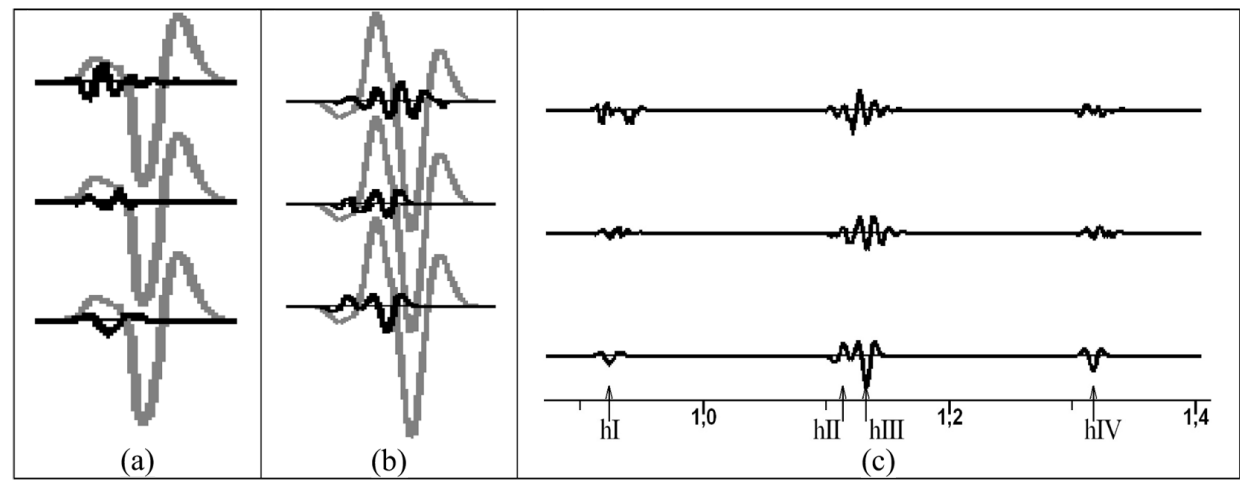

Figure $\mathbf{6}$ - Prony filtering results for individual signals and the entire trace which are provided by two different shot-time intervals. In the upper part the results correspond to the interval (1). In the middle part the results correspond to the interval (2). In the bottom part initial signals and the trace are presented.

are more consistent results and better recovery of the waveform. Thus we can conclude that in case of the optimal choice of the interval length and in the absence of noise the target high frequency components can be determined rather accurately.

We shall make a remark related to the choice of the interval duration in the construction of the Prony spectra, which are supposed to be used later in filtering in order to separate specific components. Our experience shows that the duration of such intervals is related to the main frequency $f_{S}$ of the separated component described by the ratio of $T=3 \cdot f_{S}^{-1}$. In a sense, this heuristic observation is quite clear as to the damped sinusoid there an engineering rule of three periods is applied, which provides a sta- ble definition of the damping parameter. The indicated ratio for the duration of the decomposition Prony interval can also be interpreted as an analogy of the relation of frequency and scale in the wavelet analysis. However, it should be emphasized that this is not a strict rule. Depending on the structure of the separated and overlapping signals the optimum size of $T$ may be slightly smaller or larger than the specified value. The optimum choice of the interval duration, and filter parameters will be analysed later.

\section{Constructing Prony images for time sections}

We now turn to the results of the Prony filtering that apply to all modelled section where the traces $y(x, t)$, except for signal 
component, also contain noise. The experiments were performed for different signal-to-noise ratios ranging from 1 to 5 . To illustrate we shall consider only two of them. Other experiments gave similar results. The model data used is shown in Figures $3(b)$ and 3(c). We denote the corresponding experiments as A and B.

Considering the conducted experiments as a kind of a model situation for processing real data, we shall carry out the basic elements of Prony filtering technology (Mitrofanov \& Priimenko, 2011). Initially, based on the results of standard spectral analysis, we shall select main frequency $f_{S}$ of the separated component. The values of the amplitude spectra shown in Figures $3(b)$ and 3 (c) show that the main frequencies of the analysed wave fields are in the range $(10,30) \mathrm{Hz}$. That is why the frequency of $40 \mathrm{~Hz}$ will be interesting to analyse due to the following reasons:

- it exceeds the range of the main frequencies, has a rather high intensity and is near the high-frequency part of the spectrum, which is of interest to investigate.

- Also, this frequency is the main frequency for one of the Ricker wavelets.

Thus, one of the possible filter frequencies was selected. Note that when processing real data similar arguments are usually used.

The next element is the determination of the optimal parameters for the selected frequency. They are: the duration of the estimation interval of the Prony parameters of $T$, and intervals of selection at the frequency $\Delta f$ and damping $\Delta \alpha$. As a rule, the selection of the specified parameters is carried out in a dialogue with the visual analysis of the filtering results. The quality criteria of the results are formulated on the basis of the set seismic or geological goals. Therefore, the criteria for determining the optimal parameters are quite subjective and dependent on the experience of the researcher and the range of goals. On the basis of this observation we shall mention common methodological approaches, which are used in determining the filtering parameters. They are illustrated in Figures 7 and 8, where are presented some filtering results obtained when choosing the parameters for the extraction of the wave field signal component with the main frequency of $40 \mathrm{~Hz}$. Figure 7 shows the results of the Prony filtering obtained in the experiment $\mathrm{A}$ for the data having a large signal-to-noise ratio. Based on the values of the parameters shown in the figure we shall make the following conclusions:

1. Choosing only small values of the damping parameter of $\Delta \alpha$ reduces the resolution of the resulting wave pattern (compare the Figs. 7(a) and 7(b)). This fact is also con- firmed by a comparison of Figures 8(a) and 8(b) where the results of processing the experimental data $B$ are presented.

2. The width of the frequency interval $\Delta f$ must be such as to ensure a sustained recovery of signal components for selected frequency and to "capture" minimally the field components related to other frequencies. Usually, the standard spectra serve as prior information here. Thus, in these experiments Figures 3(b) and 3(c) show that such a band should be no more than 10 or $12 \mathrm{~Hz}$. This is also confirmed by comparing Figures 7(b) and 7(c) or Figures 8(b) and $8(c)$. It can be seen that the transition to a greater band with width of $16 \mathrm{~Hz}$ brings into the wave pattern field components corresponding to adjacent frequencies, in particular, to the band of frequencies near $30 \mathrm{~Hz}$.

3. When choosing the estimation interval of $T$ it is important to use prior information about the duration of the extracted seismic signal. Thus, in the experiment considered the duration of the pulse frequency formed at $40 \mathrm{~Hz}$ was 0.07 sec (see Fig. 2(a)). When choosing a significantly higher value of $T$ there is a loss in resolution for recoverable components of the wave field. A choice of a significantly shorter duration results in either a loss of stability of the resulting estimates, or drastically reduces the number of the estimated Prony components. The latter impoverishes the recovered wave pattern, which is why the tracking of the signal in some areas of the record is lost. This is confirmed by Figure 7 (d). An attempt to improve the tracking by increasing $\Delta f$ for small values of $T$ leads only to producing "noisy" images. In the absence of prior information about the duration of the seismic signal being tested one can choose the parameter $T$ based on the relationship mentioned above between the selected frequency and the duration of the interval.

Following the selection of the parameters we obtain the final results of the Prony filtering for experiments $A$ and $B$. They are on the left side of Figures 9(a) and 9(b). Based on the objective of the processing consisting of the allocation of a model component of $40 \mathrm{~Hz}$, the final values of parameters were selected. For both experiments they were: $T=0.08 \mathrm{sec} ; \Delta f=8 \mathrm{~Hz} ; \Delta \alpha=150$. They allowed receipt of the maximum time resolution in the target signal extraction. The separated components are clearly seen at times corresponding to those that reflect the original signals in Figure 2(a). Extracting target signals constructed according to Prony images of time sections is assured even in the experiment $B$ 

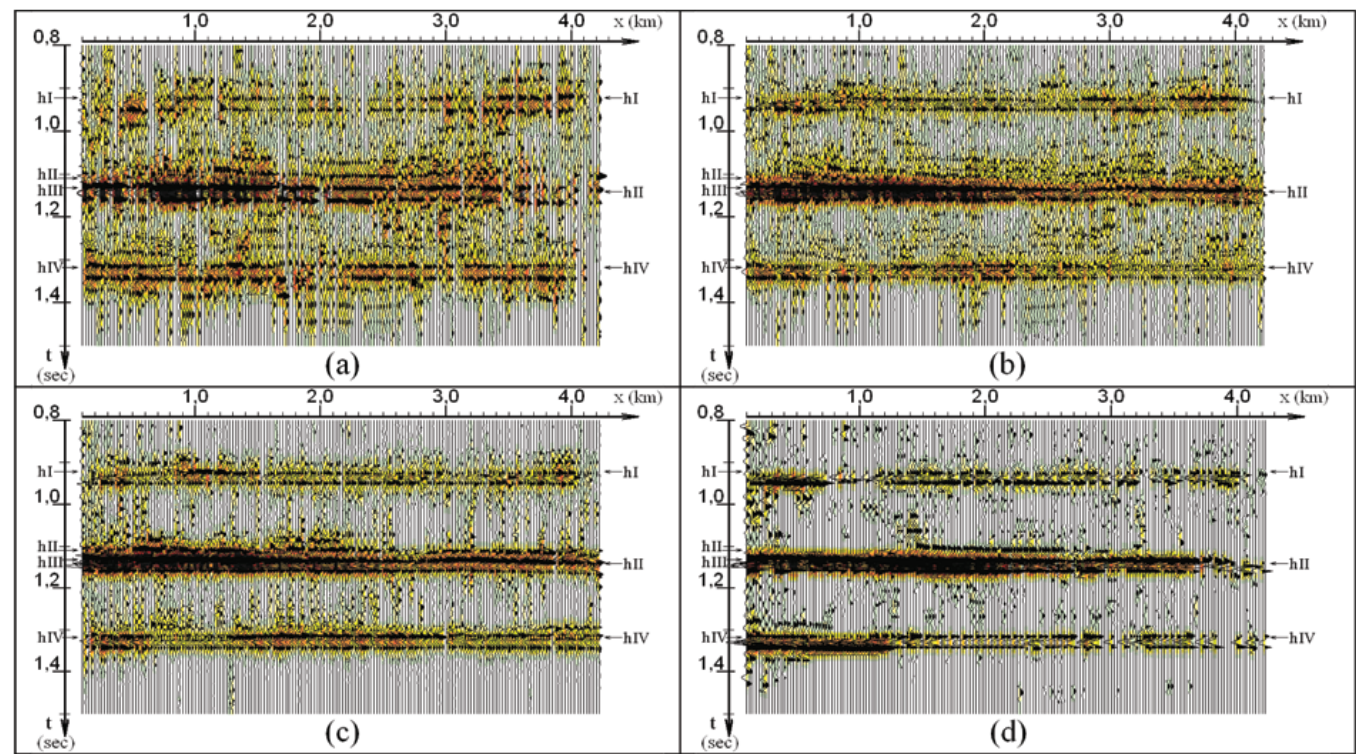

Figure 7 - Results of the Prony filtering with parameters ( $T, \Delta f, \Delta \alpha)$, used in the model experiment A: $0.2 \mathrm{sec}, 16 \mathrm{~Hz}, 20$ (a); $0.2 \mathrm{sec}$, $16 \mathrm{~Hz}, 150$ (b); $0.2 \mathrm{sec}, 8 \mathrm{~Hz}, 150$ (c); $0.05 \mathrm{sec}, 8 \mathrm{~Hz}, 150$ (d).
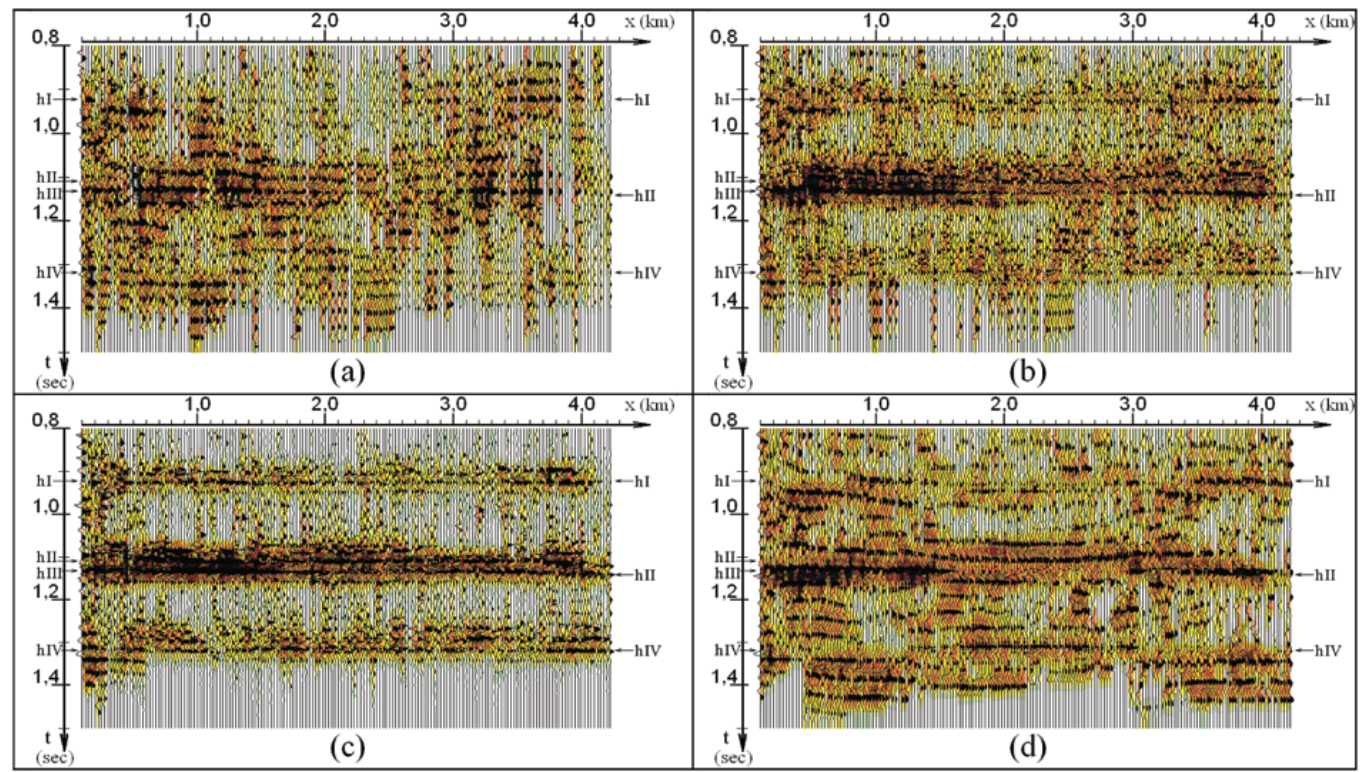

Figure 8 - Results of the Prony filtering with parameters $(T, \Delta f, \Delta \alpha)$ used in the model experiment B: $0.2 \mathrm{sec}, 16 \mathrm{~Hz}, 10$ (a); $0.2 \mathrm{sec}$, $16 \mathrm{~Hz}, 150$ (b); $0.2 \mathrm{sec}, 16 \mathrm{~Hz}, 150$ (c); $0.05 \mathrm{sec}, 16 \mathrm{~Hz}, 150$ (d).

at low signal-to-noise ratio. This indicates a high noise immunity of the method and suggests that the Prony filtering can help to obtain successful results in data processing with a signal-tonoise ratio close to 1.

We now turn to separation of a high-frequency component of the simulated wave field represented by Ricker wavelet with a frequency of $80 \mathrm{~Hz}$. During its separation one should choose new optimal parameters for Prony filtering. This is due to the fact that the optimal parameters chosen to extract the component with a frequency of $40 \mathrm{~Hz}$ may not be such for a frequency of $80 \mathrm{~Hz}$.

Following the selection of the parameters similar to what was described above we come to following values: $T=0.05 \mathrm{sec}$; $\Delta f=16 \mathrm{~Hz} ; \Delta \alpha=200$. They differ from the previous optimal parameters. These differences are in good agreement with the above general methodological techniques of choosing optimal parameters. Thus, the reduction of the range of analysis 
leads to a decrease in the corresponding signal duration (see Fig. 2(b)), and the increase in frequency and damping range leads to a more "blurred" signal spectrum and sharpening of its entry. The results obtained with the parameters mentioned are shown in Figures 10(a) and 10(b). The Prony images presented show clearly the allocated high-frequency signals relating to all the simulated horizons. In addition, we see the change of the thickness of the target horizon and its pinch point is identified well, which is associated with an increased resolution for the signal component $S^{(80)}(x, t)$.

The final results of the Prony filtering indicate an increase in the resolution of the images for the resulting sections. This allows tracking of changes in the dynamics of the target signal associated with the horizon II. Such a change, according to the model, is caused by pinching out of the horizon III. By the nature of the monitored change one can identify the horizon pinching point somewhere in the area of $3 \mathrm{~km}$. Thus, when processing similar real data one could increase the accuracy of contouring the gas reservoir. Note that the mathematical modelling programs used did not take into account the peculiarities of the seismic signal formation for the gas-containing reservoirs. The latter, as we know, has a significant effect on the absorption parameter, which gives additional benefits to Prony filtering in data processing.

As part of the experiments conducted we compared the results of the Prony and band-pass filtering. It allowed a better evaluation of the benefits of Prony filtering in the separation of the corresponding components. The results of the standard band-pass filtering are shown in the right part of the Figures 9 and 10. When the band-pass filtering was conducted we used the same band of frequencies that we used in the Prony filtering. Thus, for the results of Figure $9 \Delta f=8 \mathrm{~Hz}$, and Fig. $10 \Delta f=16 \mathrm{~Hz}$. We see that in both cases the band-pass filtering provides a lower resolution in the time variable and leads to a loss of dynamic expressiveness of the separated signals. It makes it impossible to determine features associated with pinching out of the target formation. At the same time, the transition to a greater band of frequencies used during band-pass filtering, of course, contributed to improved resolution of the allocated signals. But such increase in resolution refers only to the most energetically expressed signals components.

\section{PHYSICAL SIMULATION}

\section{Description of the physical models}

The experiments simulated the spreading process of elastic waves in media with absorption and scattering. All completed physical models are two-dimensional models. Common features for them were: a highly reflecting bottom boundary (free lower edge) and intermediate reflecting horizon in the form of a thin layer located at a depth of approximately two-thirds from the observation points to the free edge of the model. A thin layer was introduced into the model specifically as a reference in the analysis of the effects associated with the target objects. These horizons are conventionally denoted as $F$ and $A$ (for example, see Fig. 11(a)). All simulated objects were located between these horizons.

The sources and the recording system were located at the upper free edge of the model. But the registration was performed with groups of the receivers (their number in the group was 6) and a small distance of the source from the centre of the group. Thus, the wave fields of reflected longitudinal waves received in the process of simulation approximately correspond to the time sections constructed by the near offsets. Thus, time of entry of the reflected wave from horizon $A$ is about $0.4 \mathrm{sec}$, and from horizon $F$ is $0.64 \mathrm{sec}$ (for example, see Fig. 11(b)).

The target model objects can be divided into two types: the scattering and absorbing ones. The models containing scattering objects were produced on the basis of metal sheets by crosscuts through the given area. The models with an absorbing body were produced using organic glass and plastic film from which the above mentioned body was cut out. Contours of objects and corresponding parameters of depths, velocities and damping rates of absorption for the models are shown in Figures 11, 14 and 16. There were six physical models prepared. Below are the results of the processing and analysis of data for three main models.

The first of the models presented contained two objects with fractured inclusions between horizons $A$ and $F$ (see Fig. 11(a)). One object was different from another in fracture density. Fracture density in the left object was significantly higher than in the right one. The model was produced in two versions. In the first of them cracks were empty, which led to a high level of diffracted waves. In the second modification cracks were partially filled with plasticine, which reduced the level of diffracted waves generated by free cracks. Thus, the second modification of the model gave a better approximation to the real medium. So in the future we used the results of physical simulation in the second modification.

The wave pattern corresponding to this model is shown in Figure 11(b). It can be seen that the response of scattering objects appear in the wave field in the form of axes in phase balance, corresponding to diffraction from fractures, and also to change in the arrival time and the dynamics of the wave pattern of the horizon $F$.

The four other models represent different modifications of the model shown in Figure 12(a). It contained an absorbent body of 

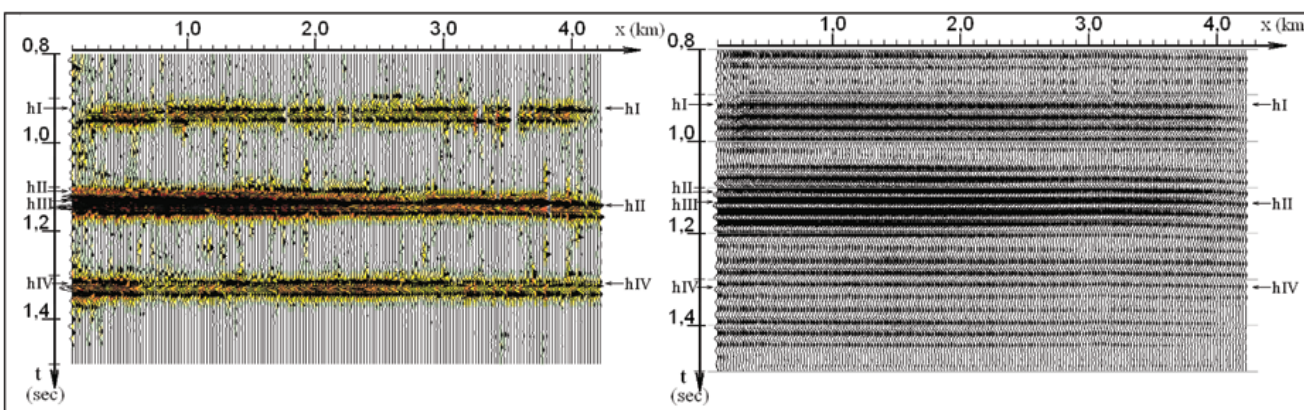

(a)
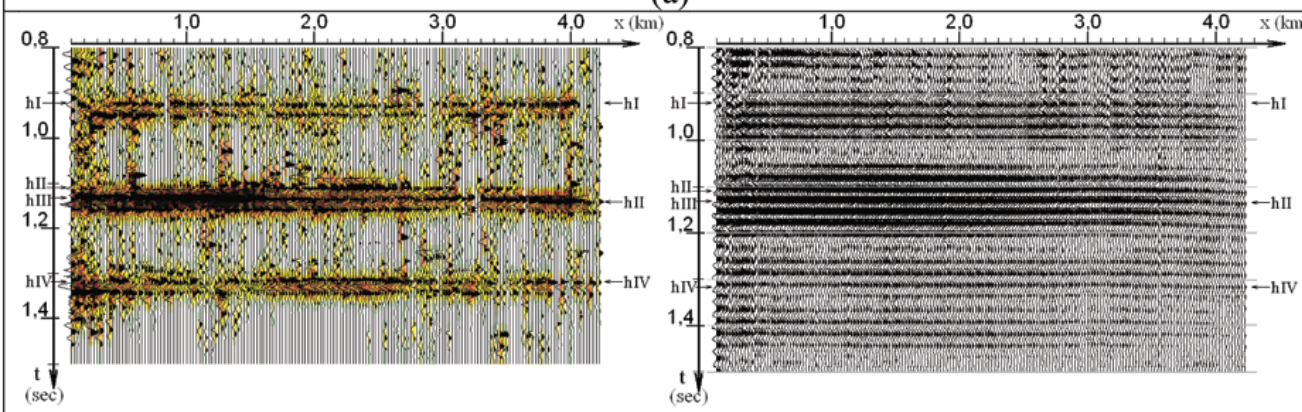

(b)

Figure 9 - Comparison of the results of Prony (left column) and band-pass (right column) filtering in the separation of $40 \mathrm{~Hz}$ signal component in experiments $\mathrm{A}(\mathrm{a})$ and $\mathrm{B}(\mathrm{b})$.
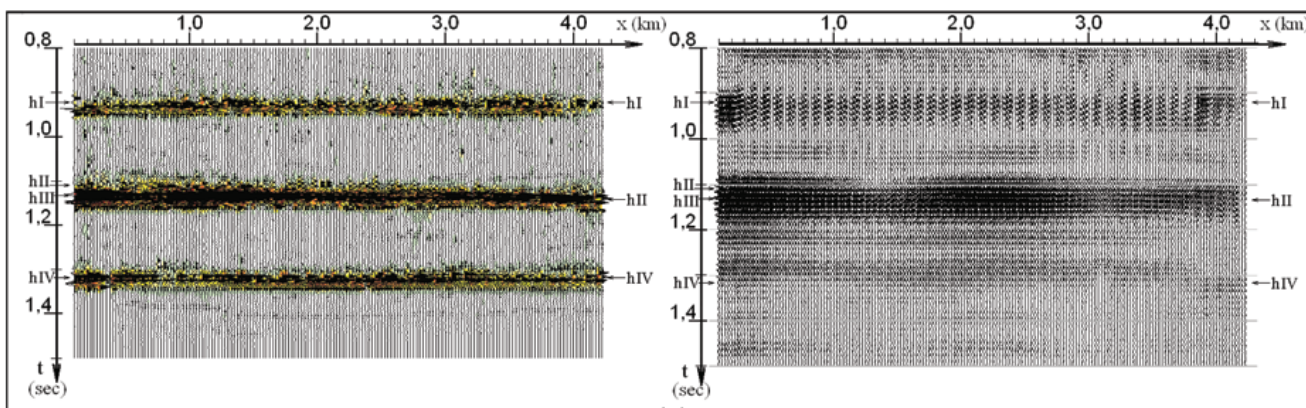

(a)
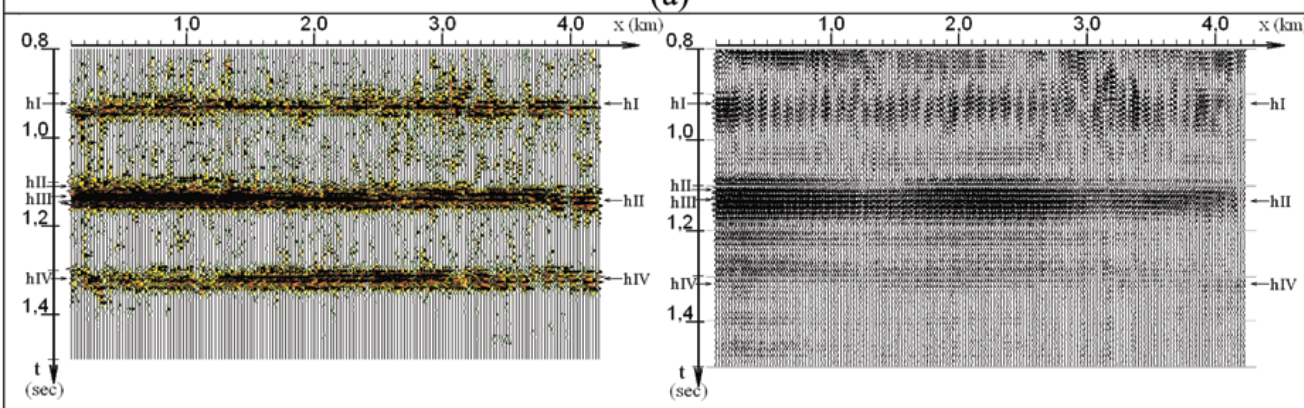

(b)

Figure 10 - Comparison of the results of Prony (left column) and band-pass (right column) filtering in the separation of $80 \mathrm{~Hz}$ signal component in experiments $\mathrm{A}(\mathrm{a})$ and $\mathrm{B}(\mathrm{b})$.

an asymmetric shape on the horizontal axis between the levels $A$ and $F$. As was mentioned above, the body was made of plastic film and was pasted on a sheet of plexiglass, which was the basis for the model. The wave pattern corresponding to the considered model is shown in Figure 12(b). The presence of an absorbing object in this case led to a slight increase in the arrival times and a 


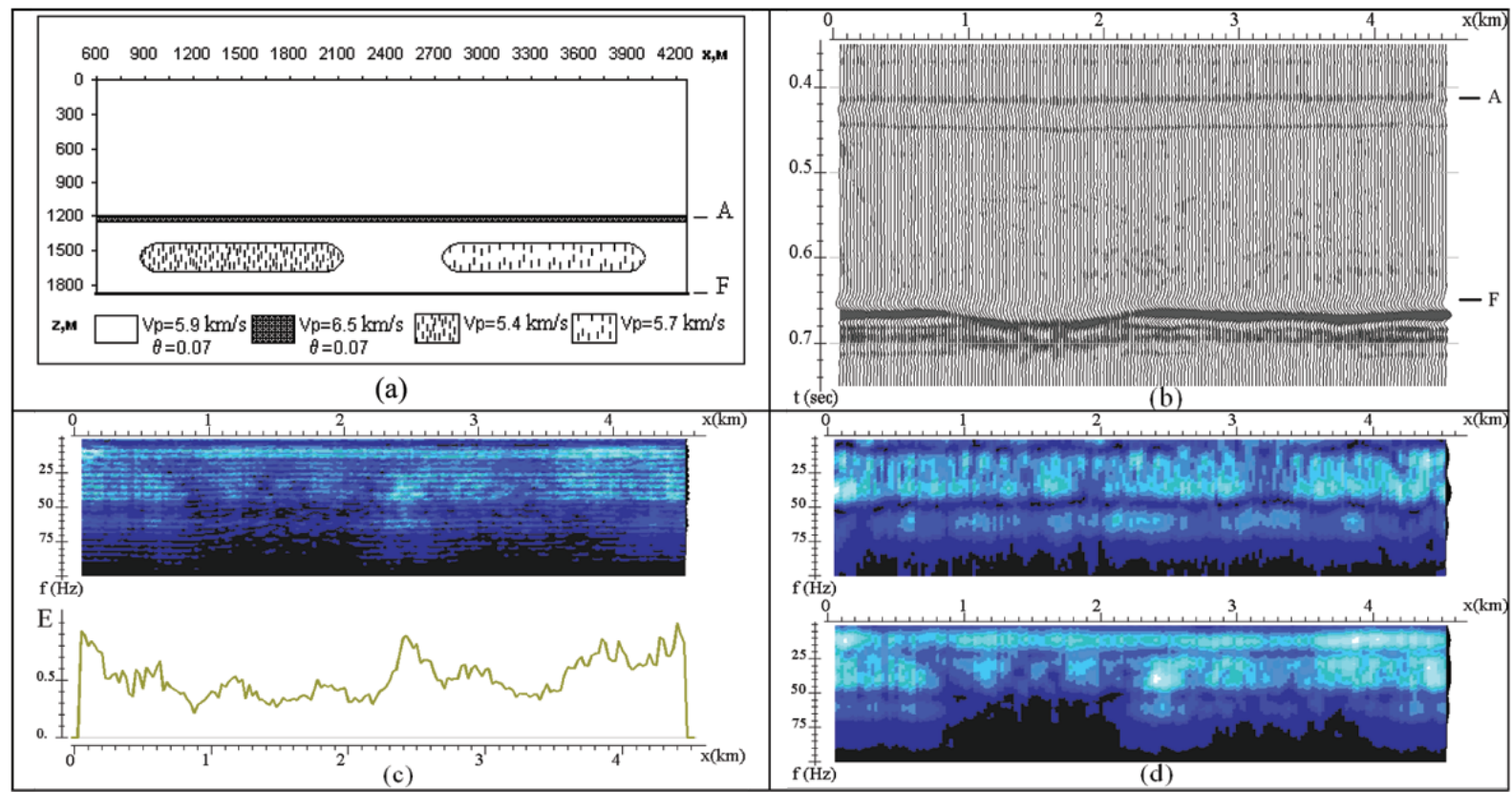

Figure 11 - Physical model with two scattering objects: its structure (a); wave pattern (b); general spectrum and amplitude (c); spectra corresponded to the reflections from horizons $A$ and $F(d)$.

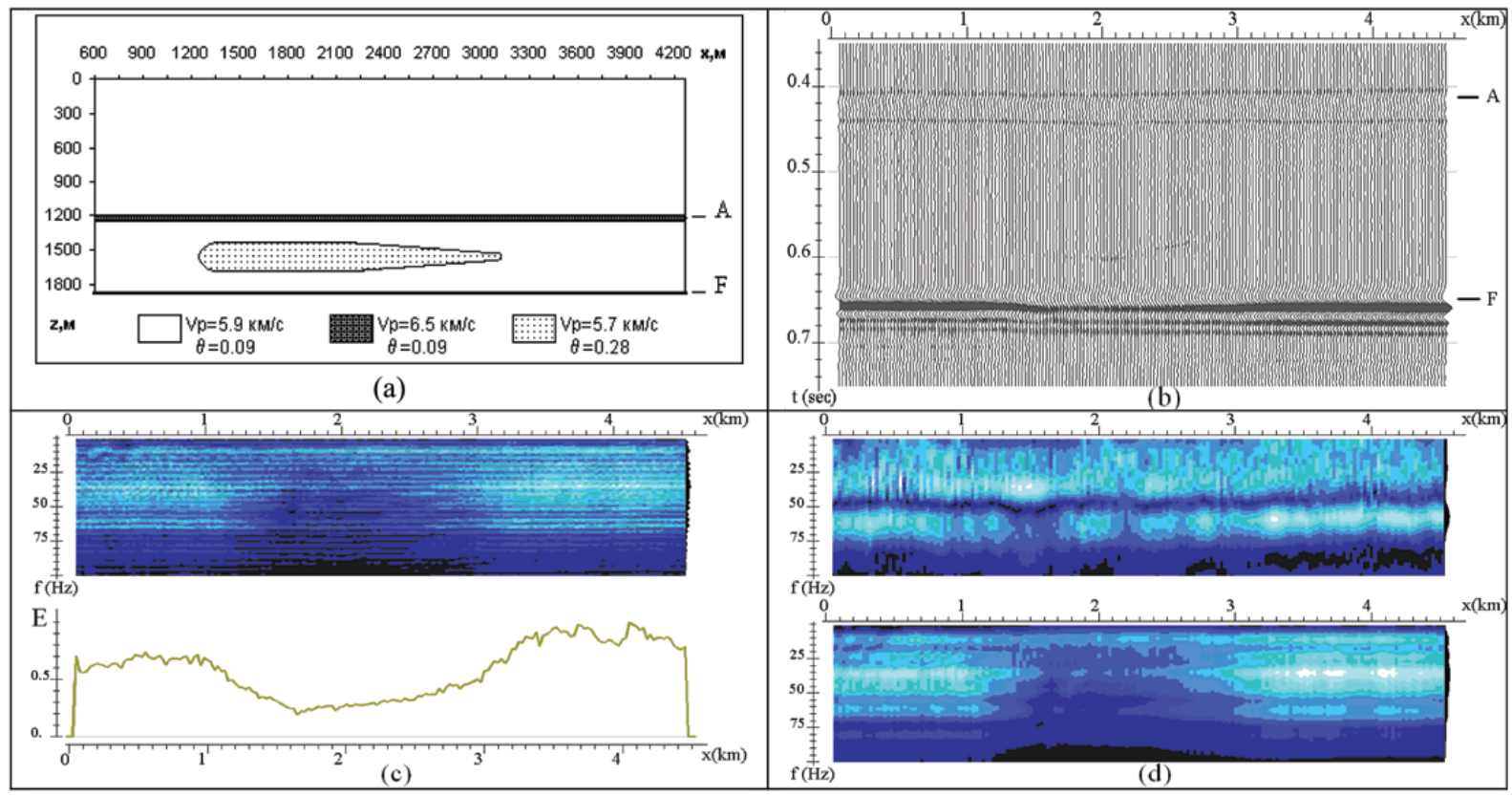

Figure 12 - Physical model with an absorbing body: its structure (a); wave pattern (b); general spectrum and amplitude (c); spectra corresponded to reflections from horizons $A$ and $F(d)$.

significant decrease in the amplitude of the reflection corresponding to the horizon $F$ under the object. The object itself in the initial wave field almost does not appear.

The subsequent models were a sort of a complication of the model shown in Figure 12 when we added four intermediate hori- zons (between horizons $A$ and $F$ as shown in Figure 13(a)). This complication of the model brought it closer to the real media and, to some extent, made it possible to assess the effectiveness of the use of the Prony filtering when studying objects in layered media. At the same time, the need to simplify the manufacture of 


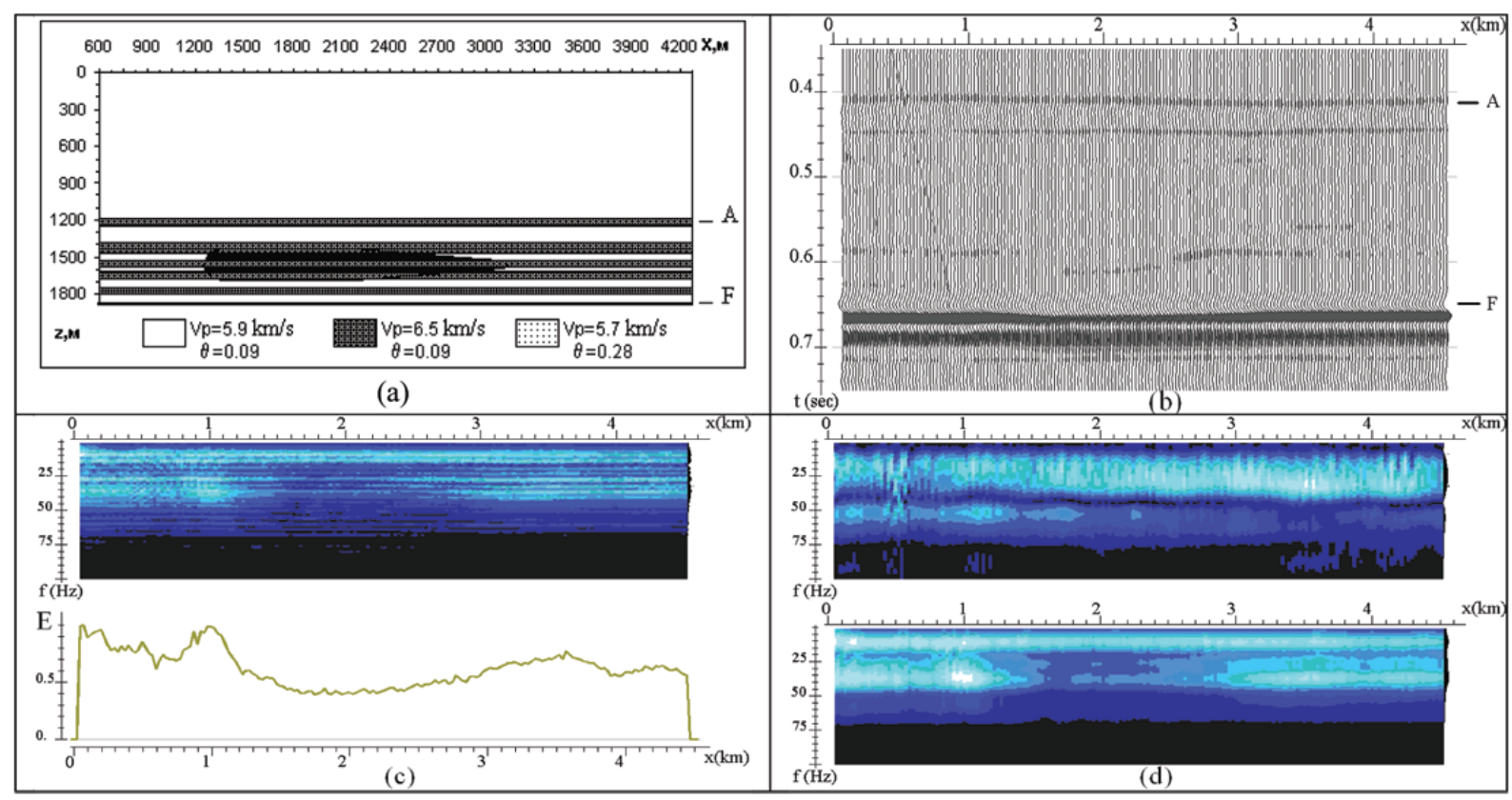

Figure 13 - Physical model with an absorbing body in a layered media: its structure (a); wave pattern (b); general spectrum and amplitude (c); spectra corresponded to the reflections of the $A$ and $F$ horizons (d).

models, which consisted of attaching an object to the side of the sheet containing the intermediate horizons or to the side, which did not contain them, did not allow us to conduct this study in full.

The most characteristic of the latest models, in our opinion, was the one where the absorbent object was attached over the intermediate boundaries. For it we managed to get a better interaction of the boundaries with the absorbing body. So below we present the results for this model. Though even with this realization we could not guarantee full and uniform interaction between intermediate horizons and the absorbing body.

The wave pattern corresponding to the physical modelling performed is shown in Figure 13(b). It can be seen that the wave field for the model under consideration is more complex than for one absorbing body. The field contains reflections associated with intermediate boundaries and the lower edge of the absorbent body. At the same time the influence of the absorbing object on the wave field of the bottom reflector $F$ decreases.

\section{The result of physical simulation data processing}

The wave fields for all models mentioned above, constructed during physical simulation, were processed on the basis of the Prony filtering technology. The following results for the three models mentioned demonstrate the main effects found during the researchers conducted.
The research of the physical simulation results started with a standard spectral analysis. Its results are presented in Figures 11 , 12 and 13. In the process of spectral analysis spectra and energy were calculated for the entire target time interval, containing the reflections analysed from horizons $A$ and $F(0.35,0.75)$ sec as well as the spectra of the individual intervals with reflections from the horizon $A(0.38,0.48)$ sec and the horizon $F(0.63,0.74)$ sec. The corresponding spectra are shown in parts (c) and (d) of the Figures $11,12,13$. Their analysis allows us to study the changing dynamics of the wave fields observed and identify possible frequency values for filtering. In particular, the results of the filtering presented clearly show the influence of scattering and absorbing objects, which were present in the models on the process of emerging reflections from the lower horizon $F$.

Then, similar to processing of mathematical simulation data we chose optimal parameters for the Prony filtering procedures. Note that when processing physical simulation data there was a significant difference in determining the frequencies of extracted signal components. If in case of mathematical simulation they were defined by a given signal component, in the case of physical models their choice is not so elementary. Just like when processing real data, it required consideration of a large number of different frequencies. This frequency selection made it possible to define a set of frequencies at which the extracted signal components allow the most accurate description of the object of study. 
All the figures below show the results of Prony filtering with optimal, in our opinion, parameters for the corresponding frequency. The parameter values as a set of $(T, \Delta f, \Delta \alpha)$ are indicated in the figures. In determining the goal was to allocate the scattering and absorbing bodies. The latter led to the fact that the main horizons $A$ and $F$ were not always determined optimally, although for other values of the parameters we could separate the specified horizons with high quality and analyse the features of their wave field. In particular, at high frequencies we could trace the changes in the structure of the wave field on the horizon $A$ associated with the unequal attachment of the thin layer.

As was already mentioned, the models investigated, despite their simplicity, differed significantly from the above examined mathematical model. First of all, it is a prior difficulty to imagine how the wave field will react to the simulated objects. In this respect, they are close to the real data. Secondly, the problem of extraction of a fixed component of the wave field cannot be strictly formulated in its research beforehand. In fact, this was the purpose of processing: to find those components of the wave field, which reveal the features of the analysed model the most clearly. Third, the high-frequency components of the field which, when processing the field data, contain the essential information on the absorbing properties of real media, do not adequately reflect the structure of a particular two-dimensional physical model due to the fact that they propagate in it as in a very heterogeneous threedimensional model.

Therefore, the selection of optimal Prony filtering parameters for each of the models under consideration simultaneously solved two problems. The first - the definition of the frequency components of the wave field, which give a better idea of the structure and properties of a specific physical model. The second - the determination and the best separation of those frequency components of the wave field, which optimally represent target objects due to structural and frequency-dependent effects.

We now describe the results obtained when processing each of the models presented.

The model with scattering objects. As follows from the results of standard spectral analysis (see Figs. 11(c) and 11(d)), the influence of the simulated scattering objects starts at the frequency of $30-40 \mathrm{~Hz}$. Therefore, Figure 14(a) shows the results of Prony filtering obtained for the main frequency of $40 \mathrm{~Hz}$. Considering the figure one can conclude that at this frequency the right scattering object with a lower fracture density is shown better. This is probably defined by the object top edge structure. With an increase in frequency up to $55 \mathrm{~Hz}$ (Fig. 14(b)) the left scattering object begins to appear brighter. Such behaviour of the objects at the specified frequencies meets the characteristics of the amplitude spectra calculated for a wide time interval. One can clearly observe here the amplification of the effects associated with the left scattering object for frequencies exceeding $50 \mathrm{~Hz}$. These peculiarities are evident on the results of the filtering in the structure of the entire wave field as well as in the form of reflection from the horizon $F$. If at the frequency of $40 \mathrm{~Hz}$ we can observe a relatively small change in the amplitude and time, then in case of the frequency of $55 \mathrm{~Hz}$ these changes become significant.

For comparison, the right sides of Figure 14 show the results of the standard band-pass filtering, when the frequency band was affected by frequency selection used in the Prony filtering.

We see that the band-pass filtering gives a lower time resolution. It only allows accurate determination of the area of energy decay of the horizon $F$, corresponding to the left object at high frequencies. Even an increase in the width of the band up to $14 \mathrm{~Hz}$ (see Figs. 14(c) and 14(d)) does not provide a significant improvement in the resolution of the horizons. At the same time Prony filtering defines almost precisely the contour of the left object, allocating its upper edge at frequencies of $55-60 \mathrm{~Hz}$ well enough.

The transition to higher frequencies (see Fig. 14(d)) does not improve the selection of the objects under consideration. Such a transition even leads to some loss of stability in the recovery of signal components. The exception is the left edge of the left object the contour of which can be refined on the basis of the result obtained. Thus, for this simulation experiment 55 and $60 \mathrm{~Hz}$ can be considered as the optimal frequencies for extracting a fractured target object. We note that the obtained effect of the top edge determination of the fractured objects was observed and used by us during the study of real carbonate bodies.

The model with an absorbing body. In this case, based on the spectral analysis results we can select three frequency bands where the change in the structure of the spectral composition of the observed wave field takes place (see Fig. 12(d)). They are the frequencies related to the following intervals: $(10,25) \mathrm{Hz}$, where the effect of the simulated absorbing object is almost absent; $(35,55) \mathrm{Hz}$, where it is rather expressed in the presence of other intensive frequencies; and $(60,75) \mathrm{Hz}$, where this influence is significant, but the intensity of all frequencies is reduced. The conducted selection of the Prony filtering parameters in the indicated bands allowed us to select four main frequencies: $20 \mathrm{~Hz}, 36 \mathrm{~Hz}, 48 \mathrm{~Hz}$ and $60 \mathrm{~Hz}$, which correspond to the respective range of frequencies and provide the opportunity 


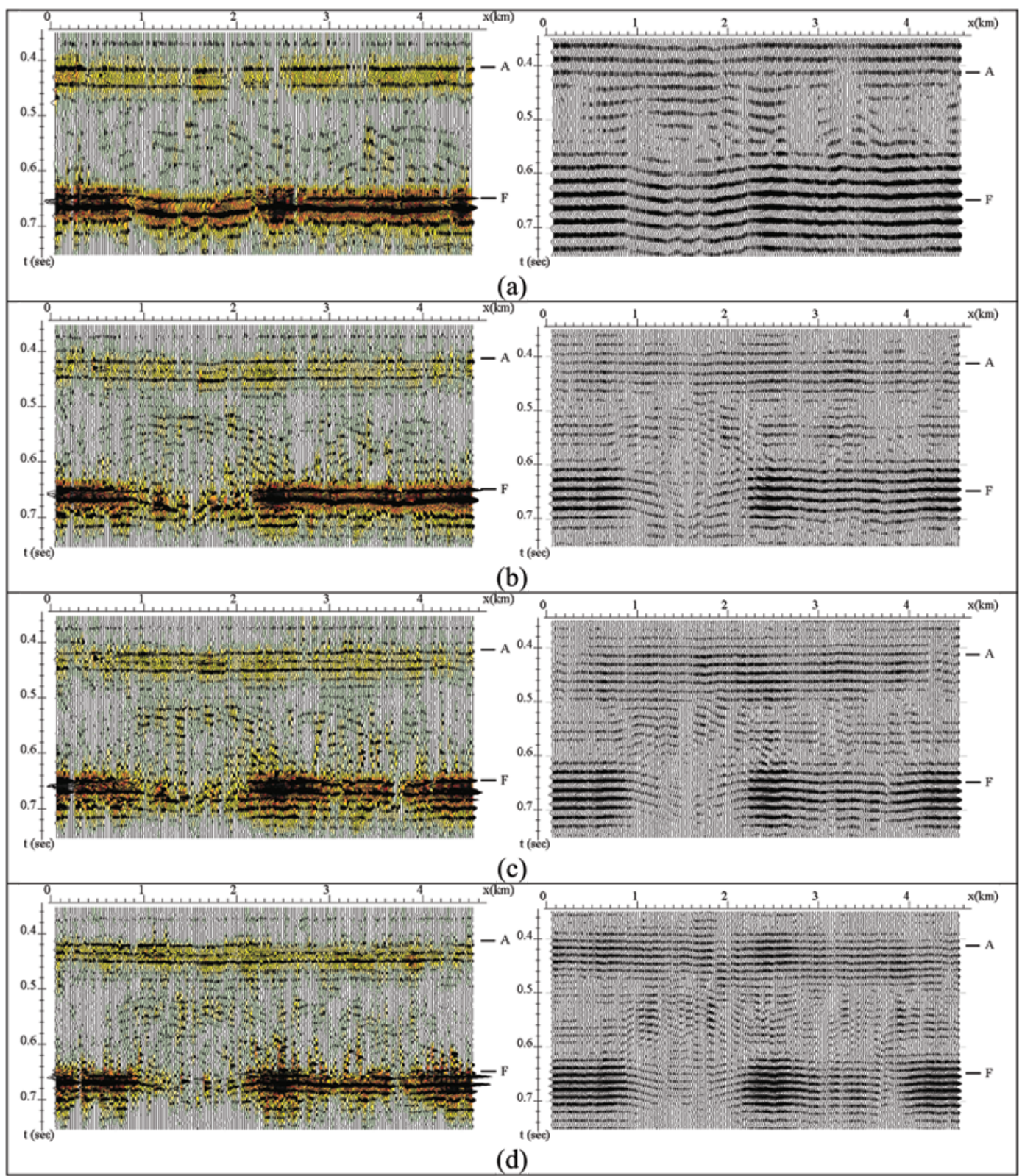

Figure 14 - Results of the Prony (left column) and band-pass (right column) filtering for a model with scattering objects at frequencies and parameters $(T, \Delta f, \Delta \alpha): 40 \mathrm{~Hz},(0.07,8,90)(\mathrm{a}) ; 55 \mathrm{~Hz},(0.07,12,200)(\mathrm{b}) ; 60 \mathrm{~Hz},(0.07,14,200)$ (c); $75 \mathrm{~Hz},(0.056,14,200)$ (d).

to explore a target object. The results obtained are presented in Figure 15.

At a frequency of $20 \mathrm{~Hz}$ the upper edge of the object is allocated as quite stable (see Fig. 15(a)). During the transition to the frequency of $36 \mathrm{~Hz}$ (see Fig. 15(b)), one can observe a structural feature of the object corresponding to a narrowing of its right side of the body. Here one can also clearly see the change in the dynamics of the horizon $F$ and there is some increase in damping in the area of the body. The transition to higher frequencies (see
Figs. 15 (c) and 15 (d)) slightly improves the delineation of the lower part of the body and the absorption region of the horizon $F$, and the inside the object. But this does not significantly increase the information about the structure of the object. Probably, such a result is due to the peculiarities of wave propagation in a two-dimensional physical model, as was mentioned above. Another explanation for this fact may be over simplicity of the model. In the analysis of this model 36 and $48 \mathrm{~Hz}$ were considered as the optimal frequencies. 


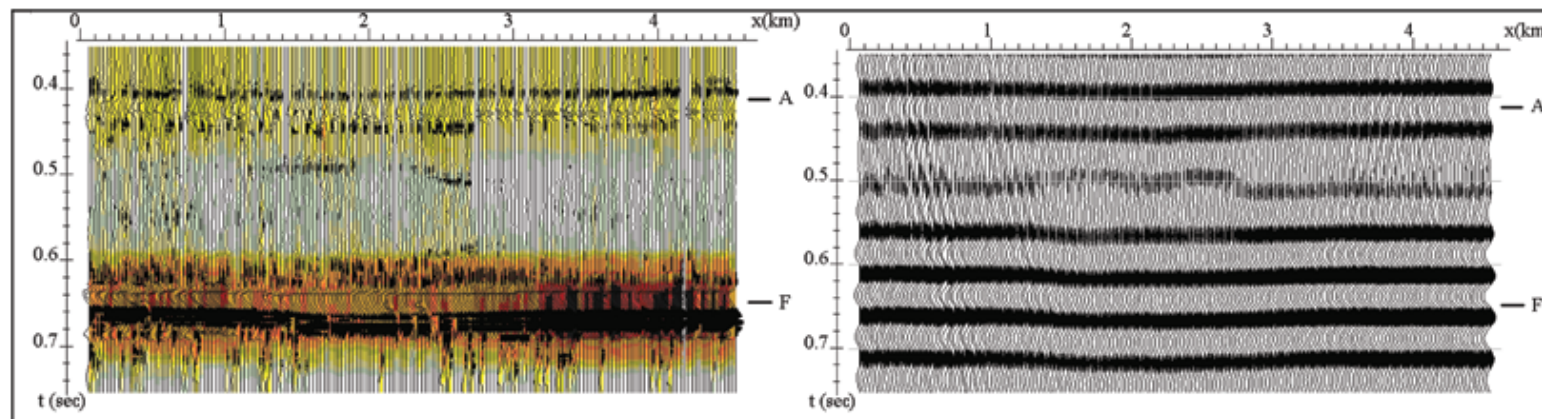

(a)

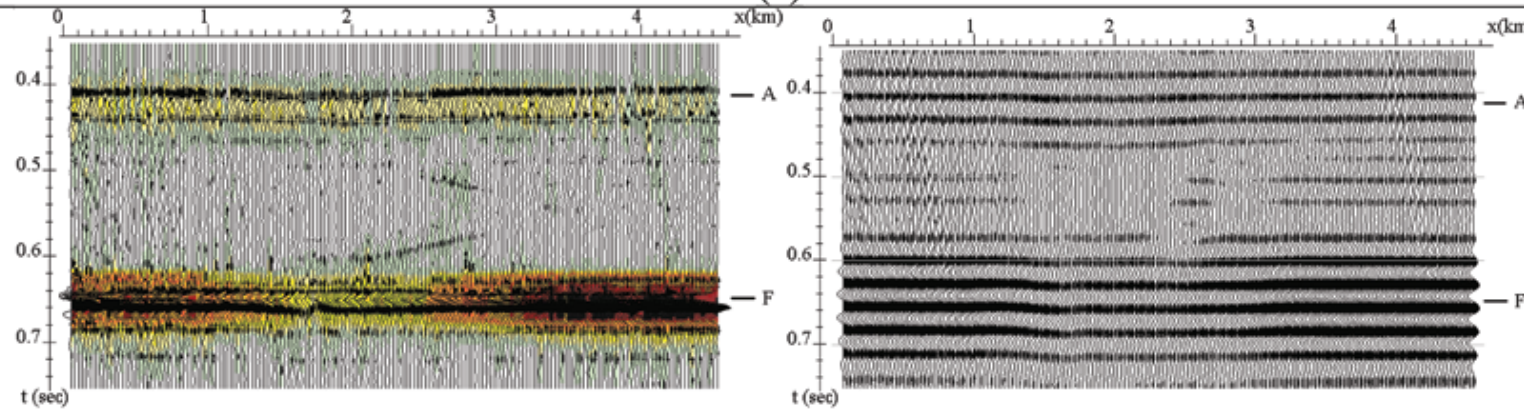

(b)

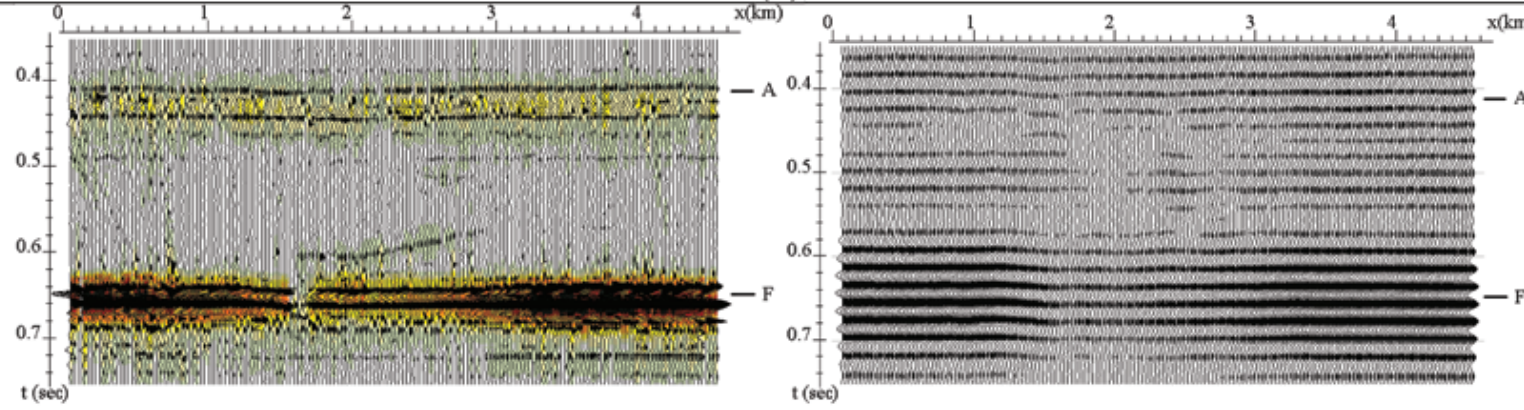

(c)

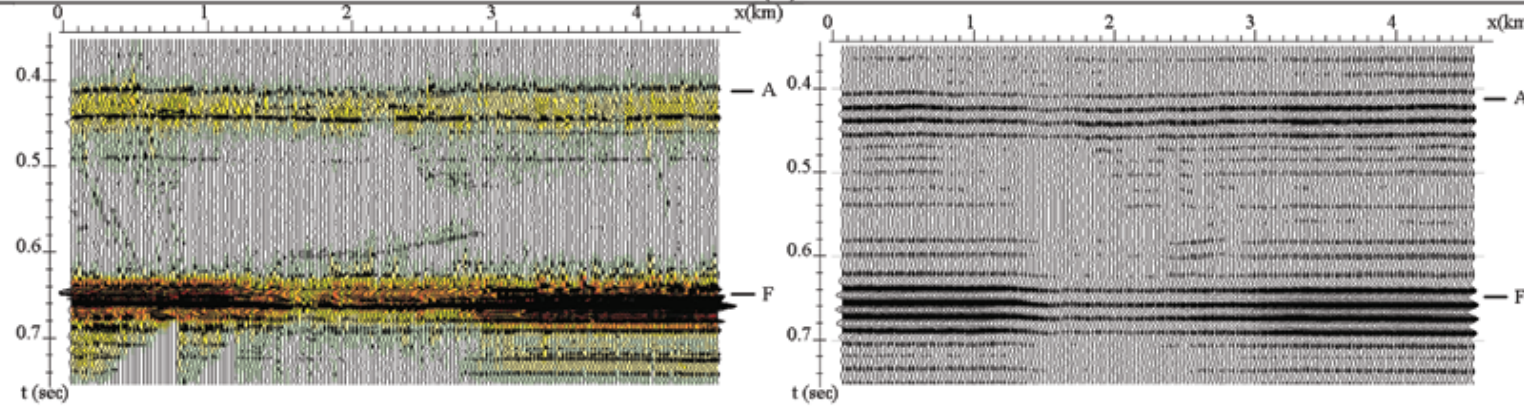

(d)

Figure 15 - Results of the Prony (left column) and band-pass (right column) filtering for a model with an absorbing body at frequencies and parameters $(T, \Delta f, \Delta \alpha)$ : $20 \mathrm{~Hz},(0.09,6,90)(\mathrm{a}) ; 36 \mathrm{~Hz},(0.08,10,90)(\mathrm{b}) ; 48 \mathrm{~Hz},(0.07,10,90)(\mathrm{c}) ; 60 \mathrm{~Hz},(0.048,20,150)$ (d).

Band-pass filtering in this case, as in the previous one, does not provide sufficient time resolution; even during the transition to a relatively wide band of frequencies of $20 \mathrm{~Hz}$ (see Fig. 15(d)).
The model of an absorbing body in a layered media.

The results of the spectral analysis show that the band of the relevant frequencies for the data is narrowed compared to the previous model. It does not exceed $65 \mathrm{~Hz}$, and stable frequency 
does not exceed 55-56 Hz (see Fig. 13(d)). The analysis conducted also shows that the most significant frequencies are located in the range of $(30,35) \mathrm{Hz}$. Therefore, we considered $25 \mathrm{~Hz}$, $30 \mathrm{~Hz}, 32 \mathrm{~Hz}$ and $56 \mathrm{~Hz}$ as the main frequencies.

The results obtained after the selection of parameters and Prony filtering at the mentioned frequencies are presented in Figure 16. It can be seen that the optimum for the study in this case is the frequencies of $32 \mathrm{~Hz}$ and $56 \mathrm{~Hz}$. The first allows us to clearly identify the layered structure of the model, and the second - very clearly reveals the effect of the absorbent body. The latter, in contrast to the model with only one absorbing body, is clearly manifested at high frequencies due to the fact that the layered media creates at high frequencies a more intense background of reflected waves outside of the object. Similar effects were observed when processing real data where the absorbent body was more evident in the medium containing additional reflecting objects near the target object. This creates the effect of "the contrast outline" surrounding the target object, which helps in the process of its allocation.

In the study of the latter model band-pass filtering was not conducted since it was obvious that it will not provide good results here. This is due to the fact that the thin layers of the media model result in a significant number of responses in the total wave field. Such responses in case of narrowband filtering will be stretched in time representation, creating a complex wave pattern with poor resolution.

\section{CONCLUSION}

The research of Prony filtering method performed in the simulation experiments allowed us to explore its features and clarify the range of problems, where the method can be used effectively. They show that if the real data (observations of the wave field) contains high-frequency components corresponding to seismic objects, then the Prony filtering allows us to identify them quite accurately. In this case the objects allocated in the wave field (for example, reflections from fixed horizon) can have rather good resolution in the temporal and spatial coordinates. During filtering process there can be changes in the shape of the extracted signal leading to its tension as well as to the appearance of additional sharp distortions. At the same time, the methodological techniques developed for determination of the optimal filtering parameters allow us to improve the accuracy of signal components extraction. Comparison of the results of Prony and band-pass filtering demonstrates significant advantages of the first over the second in the separation and analysis of signal components with different frequencies. This provides a good opportunity for the method to determine the frequency sensitive effects and localization of regions with different damping/dispersion of seismic energy. The results

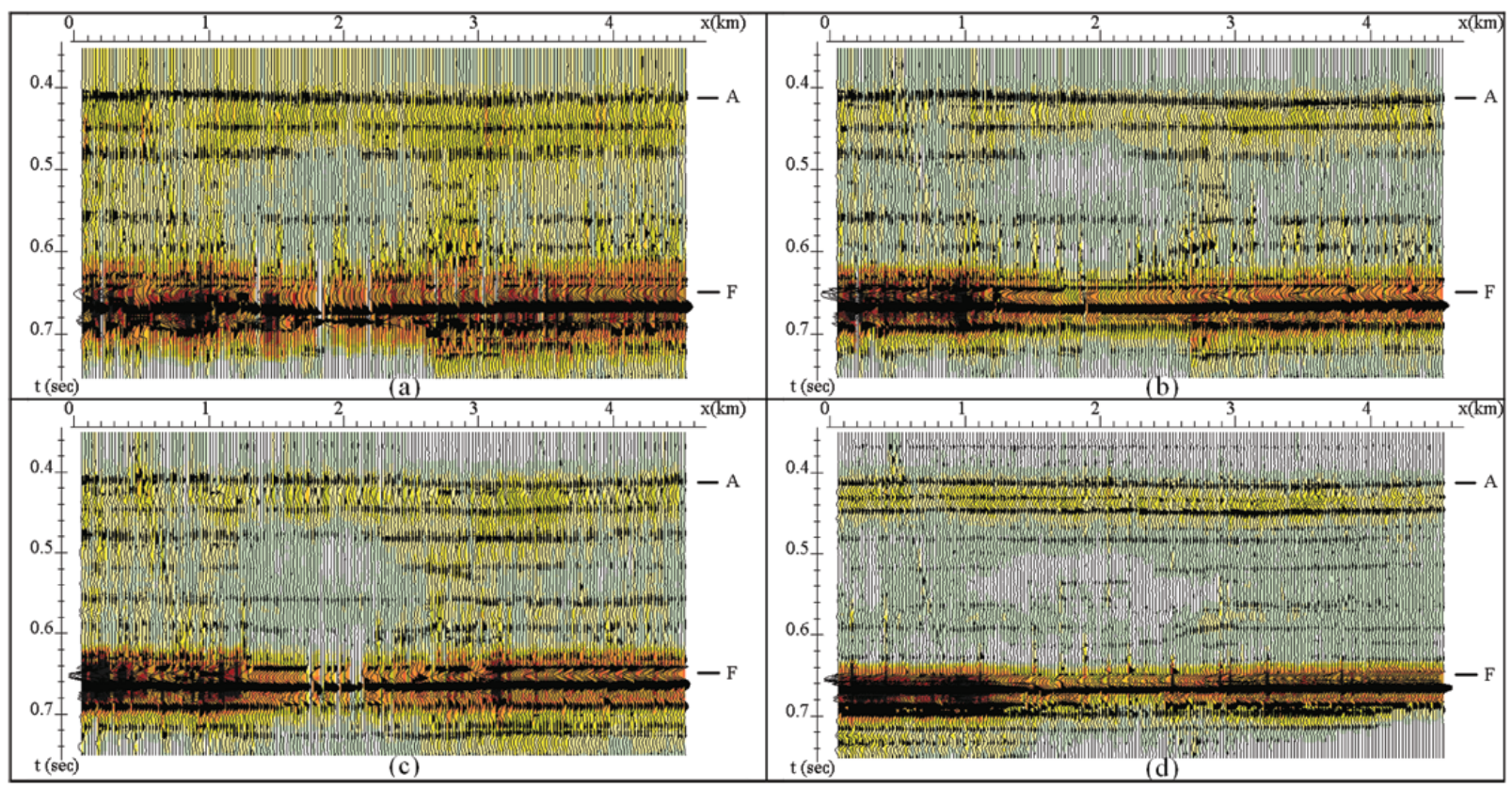

Figure 16 - Results of the Prony filtering for a model with an absorbing body in a layered media at frequencies and parameters $(T, \Delta f, \Delta \alpha): 25 \mathrm{~Hz},(0.09,8,90)(\mathrm{a})$; $30 \mathrm{~Hz},(0.09,8,90)(\mathrm{b}) ; 32 \mathrm{~Hz},(0.07,10,150)(\mathrm{c}) ; 45 \mathrm{~Hz},(0.05,12,150)(\mathrm{d})$. 
obtained also demonstrate a rather high noise stability immunity of the method, which allows it to be used when dealing with the seismic data, where the signal-to-noise ratio is equal to or greater than 1. Application of the method in the processing and analysis of the results of physical simulation demonstrated its ability of examining fractured objects and areas of the media with high absorption of seismic energy.

\section{ACKNOWLEDGMENTS}

The authors wish to express their gratitude to PETROBRAS and INCT-GP/CNPq/MCT, Brazil, for the technical and financial support for this study. The first author is especially thanks to the North Fluminense State University Darcy Ribeiro, RJ, Brazil, for providing support as a visiting researcher in LENEP/CCT/UENF.

\section{REFERENCES}

KLEM-MUSATOV KD \& AIZENBERG AM. 1984. The ray method and the theory of edge waves. Geophysical Journal International, 79(1): 35-50.

MITROFANOV GM \& PRIIMENKO V. 2011. Prony filtration of seismic data: theoretical background. Revista Brasileira de Geofísica, 29(4): 703-721.

MITROFANOV GM, ORLOV YA, RAKHMENKOULOVA IF \& KURDYUKOVA TV. 1999. Testing of the Proni filtering on the results of model data. In: Technical Programme $61^{\text {st }}$ EAGE Conference and Technical EXhibition, 7-11 June 1999, Helsinki Fair Centre, Finland, Expanded abstracts. CD-ROM.

Recebido em 26 fevereiro, 2013 / Aceito em 17 maio, 2013

Received on February 26, 2013 / Accepted on May 17, 2013

\section{NOTES ABOUT THE AUTHORS}

Georgy Mitrofanov graduated in Geology and Geophysics, Novosibirsk State University, Akademgorodok, Novosibirsk, Russia, in 1972. He received his Ph.D. in Mathematical Physics in 1984. He is currently a visiting professor of the Laboratory of Petroleum Engineering and Exploration, North Fluminense State University Darcy Ribeiro, Macaé, RJ, Brazil. Areas of interest: seismic data processing, direct and inverse problems in geophysics, reservoir characterization.

Viatcheslav Ivanovich Priimenko graduated in Applied Mathematics and Mechanics, Novosibirsk State University, Akademgorodok, Novosibirsk, Russia, in 1978. He received his Ph.D. in Mathematical Physics in 1990. He is a professor of the Laboratory of Petroleum Engineering and Exploration, North Fluminense State University Darcy Ribeiro, Macaé, RJ, Brazil. Areas of interest: direct and inverse problems in geophysics and petroleum engineering, numerical modeling and migration. 